QUARTERLY OF APPLIED MATHEMATICS

VOLUME LXIX, NUMBER 2

JUNE 2011, PAGES 291-308

S $0033-569 X(2011) 01220-3$

Article electronically published on March 9, 2011

\title{
MODELING CONSCIOUSNESS AS AN ADAPTIVE PHENOMENON
}

\author{
BY \\ WILLARD L. MIRANKER \\ Department of Computer Science, Yale University
}

\begin{abstract}
We start at the level of a bacterium where an observer feature associated with foraging measurements motivates introduction of a notion of awareness (a protoconsciousness) as a primitive, a dualist construct. The relative simplicity of Darwinian concepts at the level of a bacterial colony then leads to development of an analytic theory of perceptual consciousness that includes colonial notions of qualia. Results of simulations that verify the colonial principles developed are given. We extend this approach, first to a neuron and then to a neuronal assembly, each extension accompanied by a derived consciousness construct. For each construct (dualist or derived), a mathematical model in the form of a measurable quantity called a token is developed. The pairs, token and construct, permit the design of experiments that would validate the theory. Applications of the theory are developed, each based upon the analytic model (the tokens) and each explaining a familiar aspect of human consciousness in mathematical terminology.
\end{abstract}

1. Introduction. The following critique of Schrödinger [30, "A physical scientist does not introduce awareness (sensation or perception) into his theories, and having thus removed the mind from nature, he cannot expect to find it there", motivates using a dualist approach 11 to develop an analytic theory of perceptual consciousness 2 Darwinian concepts (Darwin [8): competition for nutrient, natural selection and reproduction and their $20^{\text {th }}$ century addenda are also used (Dawkins 9]), Weismann (see Mayr [22]). A principle difficulty in studying human consciousness is its complexity 3 So our development is divided into two parts. In Part I, which deals with the simpler context of

Received September 7, 2009.

2010 Mathematics Subject Classification. Primary 92-XX.

Key words and phrases. Bacterial colony, consciousness, Darwinism, dualist construct, neuronal assembly.

${ }^{1}$ By a dualist approach we mean the taking of a feature of nature as a primitive into a scientific model. Such a primitive is called a dualist construct. See Section 1.4.

${ }^{2}$ As a primitive there can be no definition of consciousness. For a working picture of perceptual consciousness, we may take the experiencing of feelings (hunger, love, fear...), qualia (colors, sounds, pain...), and the notion of self/intentionality... To explain this experiencing has been called the hard problem of consciousness (Chalmers [4]).

${ }^{3}$ For an indication of this complexity, see a varied collection of approaches to the subject: Churchland (5], Dennett [10, 11], McDermott [23], Penrose [28, Stapp [32, Velmans 35]...

(C)2011 Brown University Reverts to public domain 28 years from publication 
a model bacterium (Margulis 21]) and a model bacterial colony (Ben Jacob et al. [3], Waters, Bassler [36]), a notion of awareness (a proto-consciousness) as a dualist construct is introduced. (See Sheets-Johnstone 31 for a discussion of consciousness without nervous systems.) In Part II, which deals with a model neuron and their assemblies, a notion of consciousness as a primitive is introduced at a more complex human-like level. The elemental colonial analogs of consciousness bear only a remote resemblance to the rich phenomenology of human level consciousness counterparts that are subsequently developed. It is this simplification in Part I that is the key to getting started.

Terminology. Since we discuss the consciousness primitive in two contexts: bacterial (and colonies) and neuronal (and their assemblies), and because consciousness at the bacterial level is so elemental compared to the neuronal, we shall use different terms, namely awareness in the bacterial context and consciousness in the neuronal.

1.1. Outline of Part I (Sections 2 and 3). We begin Part I in Section 2 with the development of the bacterial case by modeling chemotaxis, the locomotion of bacteria based upon nutrient concentration measurement differentials (Curtis, Barnes, p. 131 [6]), and we introduce a notion of fitness that characterizes bacterial ability at foraging for nutrients. A virtual gene that records the fitness, that is, that records progress in the competition for survival is used. An observer feature is required to accompany a physical measurement (Stapp ([33]), and we introduce a dualist construct that supplies one (Margenau [19], 20]). Motivated by those foraging measurements, the construct is assigned the role of primitive awareness (a proto-consciousness). It is introduced in association with bacterial fitness, and its extension to colonial awareness is developed (Velmans [35]). In Section 3, we show how the dualist construct coupled with Darwinian features of competition, natural selection and reproduction enable the development of a colonial notion of qualia. It is the simplicity of features of awareness in a bacterial colony compared to human level consciousness counterparts that facilitate the development. Turing 34 observed that competition between competing tendencies (such as foraging and reproduction in our model) is basic for the emergence of complexity (such as the colonial notion of qualia). Section 3 concludes with simulations that illustrate and support the features of the model.

1.2. Outline of Part II (Sections 4 and 5). We begin Part II in Section 4 by employing neuronal features analogized from the bacterial case. The notions of foraging for nutrients, of fitness (along with an associated dualist awareness construct), and of reproduction are extended first to a model neuron and then to a neuronal assembly. To do this we introduce a number of analogies. (i) Nutrient flux is replaced by neurotransmitter flux. (See Section 4.1.) (ii) Foraging, that is, position changes in space, is replaced by position changes in synaptic weight space. This brings an increase of as much as five orders of dimension magnitude, which speaks to the complexities to be found in human consciousness. (iii) A bacteria's virtual gene is replaced by a (virtual) neuronal chromosome, the latter composed of the neuron's synaptic weights. (iv) Neuronal competition is implemented by inhibition, and reproduction in the colony is replaced by the opportunity for a neuron to fire. (See Section 4.2 for (ii), (iii) and (iv).) In Section 5, the development of a notion of qualia is carried over from the bacterial case to the neuronal 
case, so that the resemblance to human level aspects of consciousness is more transparent. (Compare the notion of Neural Darwinism and the high complexity of the dynamic core in Edelman, Tononi [12.) Applications illustrating and supporting the neuronal features developed by our model are given in Section 5. As motivation, we show that the colonial awareness introduced in Section 2 vanishes with the disappearance of nutrients. Then the applications in the following list that are relevant to human consciousness are developed. Each application suggests an experiment that contributes to the validation of the theory.

1. The inability to experience certain qualia in the absence of sensory input.

2. Answering the question: Why does the brain locate the perception of a quale (such as pain, taste, smell) in the peripheral nervous system? We confirm as well the existence of a threshold for such a perception.

3. A corollary of 2: How do visual and auditory imagery come to be located 'out there' in the surrounding space?

4. How do dream images come to be located in a seemingly virtual space?

5. Hallucinations, phantom pain.

6. The threshold process involved in unconscious sensing (pheromones).

A discussion of the way that the theory informs the evolution of consciousness concludes Section 5 .

1.3. Tokens, experimental verification. The theory makes use of an observer quality, a primitive awareness (proto-consciousness) in Part I or consciousness in Part II, each introduced as a dualist construct. Other constructs are derived as elaborations of the basic level (Llinas [18]). Each construct is complemented by a measurable physical quantity, an analytic expression called a token (Miranker (24], 26])). A token is observable but unconscious, while its corresponding construct is unobservable but conscious. For example, an experiment measuring that there is adequate strength in the value of a token makes for the theory's prediction of a feature of consciousness corresponding to the token's associated construct. That prediction could be compared to the experience of the subject. We do not know how to learn of experiences of non-human connectionist systems (biotic or abiotic), so we might apply this token/construct procedure to motivate study and speculation on their nature. (Compare Nagel 27.)

1.4. Reductionism. Just as Newton's laws of motion and gravity provide a dualist framework for predicting and measuring the phenomena and behaviors of particle dynamics (see footnote 1), so our theory is intended to provide a framework for study and validation (prediction and measurement) of consciousness and its associated phenomena. Newton's constructs, action at a distance, for example, have retained their dualist quality now for centuries. Should a reductive explanation of consciousness be found, this in the face of Schrödinger's critique, it would be a reduction to Newton's laws upon which classical science rests. Such an explanation would be reductive in the narrowest sense, if at all. 


\section{Part I: Awareness in a colony of bacteria.}

2. The bacterial model. Bacteria are imbedded in a nutrient bath, whose concentration $n(x, t)$ represents information critical to bacterial survival. To process this information, bacteria make measurements to which they respond by changing location in an attempt to improve nutrient supply (Curtis, Barnes [6]). We introduce a dynamic fitness quality $a(t)$, which records the signed performance of a bacterium in making a nutrient supply improvement 4 (Damasio [7]). We also introduce a virtual gen 5 g $g(t)$ that records fitness cumulatively and that grades the bacteria's motile behavior. (One such gene is adequate for this modeling purpose.)

2.1. Nutrient supply, measurement, colony population. To characterize motility, a bacterium is modeled as a rod-like object of length $d$. Its two ends are denoted $r$ and $l$, respectively. Let $x_{r}, x_{l}$ be the locations at time $t$ of the $r, l$ ends, resp. of a bacterium. Nutrient measurements $n(x, t)$ and $n\left(x_{l}, t\right)$ at the ends are made by the bacterium. (To complete any measurement process, we take it that an observation of the measured values must be made. This feature, central to the development of the primitive awareness dualist construct will be described in the following Section 2.2.) The difference and mean of the values of these measurements are computed:

$$
\begin{aligned}
\Delta n(t) & =n\left(x_{r}, t\right)-n\left(x_{l}, t\right), \\
\bar{n}(t) & =\left(n\left(x_{r}, t\right)+n\left(x_{l}, t\right)\right) / 2 .
\end{aligned}
$$

Motion. The change in position of a bacterium from $x(t)$ to $x(t+1)$ is modeled as a displacement proportional to $g \Delta n$ plus a rotation. To specify $x(t+1)$, first consider the rectilinear displacement (with scaling factor $\alpha$ )

$$
y(t+1)=x(t)+\alpha g z \Delta n,
$$

in the direction

$$
z=\operatorname{sgn}(\Delta n)\left(x_{r}-x_{l}\right) / d
$$

Next, let $R(\theta, \varphi)$ be the three-dimensional rotation matrix, where the rotation parameters $\theta$ and $\varphi$ are chosen uniformly at random. Then $x(t+1)$ is given by

$$
x(t+1)=R(\theta, \varphi)(y(t+1)-\bar{y}(t+1)),
$$

where $\bar{y}(t+1)=0.5\left(y_{r}(t+1)+y_{l}(t+1)\right) \cdot y(t+1), y_{r}(t+1)$, and $y_{l}(t+1)$ are computed from (2.3). So the tumbling motion of a bacterium is modeled as a jump along its length specified in (2.3) followed by a random rotation specified in (2.5).

Colony population, nutrient supply: Let $p(x, t)$ denote the number of bacteria in a colony at position $x$ at time $t$, let $\beta$ be the quantity of nutrient consumed by a bacterium in unit time, and let $n_{e}(x, t)$ be an exogenous (signed) influx of nutrient. Then

$$
n(x, t+1)=n(x, t)-\beta p(x, t)+n_{e}(x, t) .
$$

\footnotetext{
${ }^{4}$ The term 'fitness' in biology is often used to mean the ability to survive and reproduce. For reasons of compatibility, we shall view $a(t)$ as a quantitative representation (token) of this ability.

${ }^{5} g(t)$ is called a virtual gene to underscore analogies to real genes and to the genes appearing in genetic algorithms.

${ }^{6}$ Allowing more than one bacterium at a point $x$ is a technical idealization. Addressing this issue, while straightforward, would add inessential complexity to the model.
} 
2.2. Fitness token, dualist construct, awareness hypothesis and conventions. With movement, the fitness of a bacterium is taken to be

$$
a(t+1)=\operatorname{sgn}\left[\frac{\bar{n}(t+1)}{\bar{n}(t)}-1\right], \quad \text { with } \operatorname{sgn} 0=1 .
$$

We say that a bacterium is fit or not-fit at time $t$ if $a(t)=1$ or -1 , that is, if its motion (behavior) improved or degraded its nutrient supply. $a(t)$ is a signature-valued indicator of change in the bacterial nutrient supply resulting from motion. The process summarized by (2.7) is taken as an observation of the nutrient measurement values anticipated in Section 2.1. This process, taken as a dualist construct, is hypothesized to be an awareness quality (a proto-consciousness) of nutrient supply change by the bacterium 7 Motivation for this choice comes from the observation that the signs of $a(t)$ correspond to alternative responses of a bacterium to behavior favoring/disfavoring survival.

This signatory choice for $a(t)$ is not a limitation, since $g(t)$ is taken to record bacterial fitness history, namely

$$
g(t+1)=g(t)+a(t+1)
$$

and it is in terms of $g(t)$ that bacterial mobility is graded (see (2.3)). Note the correspondence between this observation/awareness notion and the one supplied by Hebb's law in the neural network consciousness model of Miranker [26.

Conventions, processes and their tokens, definition of awareness.

1. The dualist awareness process just described will be denoted by $\hat{a}$. The fitness value $a$ itself (specified in (2.7)), is termed a token 8 of the process $\hat{a}$.

2. Throughout, a token and its associated process will be given the same label, except that the latter will have a hat appended (as with the pair $(a, \hat{a})$ ).

3. Definition: A quality of being aware/not-aware is attributed to a process if its token is positive/negative (i.e., if awareness equals/not-equals $\varnothing$ (see (3.2)ff). At the bacterial level, being aware/not-aware is equivalent to being fit/not-fit. (For the motivation for this definition, see the discussions following (2.7) and (2.9).)

2.3. Population changes, causality, changes in $g$ upon reproduction. Three effects change $p(x, t)$ : (i) motion, (ii) reproduction, and (iii) competition for survival.

i) Motion. From (2.5), we see that a point $x$ in a bacterium is displaced to $x(t+$ $1)=R(y-\bar{y})$, the variables being relevant to that bacterium. While there are ways to characterize a cell's breadth, for definiteness (and clarity), we consider a bacterium as being located at its midpoint.

ii) Reproduction. Bacterial reproduction proceeds by binary fission, and the resulting daughters are assigned fitness values at random from among the four possibilities,

\footnotetext{
${ }^{7}$ Measurement and observation of the measured value is taken as a fundamental pairing in physics. This duality has entered the study of consciousness (Miranker ([25], 26]) and Stapp ([32], 33]).

${ }^{8}$ Classical mechanics supplies an analogical example of such a pair. The pressure (a measurable physical quantity) is a token of its derived construct, the specific force (the latter a concept specified in terms of the dualist constructs of mass, space and time upon which classical physics is developed).
} 
$\{ \pm 1, \pm 1\}$. However, since we do not expect a not-fit mother to divide into two fit daughters and inversely, the assignments $(1,1) /(-1,-1)$ are denied to the daughters of a notfit/fit mother. This protocol embodies a mutation effect. Of course, the fitness may change as soon as the next bacterial displacement (see (2.6) and (2.7)).

iii) Competition: survival and death. There is enough nutrient at $x(t+1)$ for

$$
s=\left\lfloor\frac{n(x, t+1)}{\beta}\right\rfloor
$$

bacteria to survive a unit time. If there are $f$ fit bacteria at $x(t+1)$, then $u=\min (s, f)$ of them survive. Bacteria that don't survive are eliminated from the colony.

An alternative selection protocol is to allow some of the not-fit to survive. In this case $s \geq f$, so that $u=f$, and then all the fit survive. Then there is enough nutrient for $v=\max (0, s-u)$ of the not-fit to survive also. Recall that fit/not-fit bacteria are aware/not-aware. If the number of aware and/or not-aware bacteria exceed the numbers $u$ and/or $v$, resp., make a random selection for the $u$ fit and/or the $v$ not-fit survivors, resp. Variations on this procedure (for example, allowing access to nutrient to depend stochastically on fitness) as well as study of such survival issues as bacterial competition for space is left for future study.

Population dynamics. Combining the effects $i$ ), ii) and iii), we have

$$
p(x, t+1)=\min \left[f, s, \sum_{x(t)}\{p(x, t)\}_{x(t+1)}\right] .
$$

Here the subscript $x(t+1)$ denotes that fraction of the $p(x, t)$ bacteria that are displaced from $x(t)$ to $x(t+1)$. $f$ is dropped from the bracket in (2.10) for the alternative selection protocol.

Causality. Reproduction is guided by fitness $a$. Taking reproduction to be guided by the awareness $\hat{a}$ attributes a causal feature to that dualist construct.

Updating the value of the gene, recording of fitness/awareness. With fission, the value of the virtual gene is taken to change as follows:

$$
g_{\text {daughter }}=g_{\text {mother }}+a_{\text {daughter }}+v \chi .
$$

In (2.11), $v$ is a scaling factor, and $\chi$ could be taken as a normally distributed random variable with zero mean. Then $v \chi$ is a mutation term. From (2.7) and (2.11), we see that the net cumulative fitness/awareness competitive reproductive history is recorded in the gene.

Referring to (ii) Reproduction, we see the tendency for fit/not-fit progeny to improve/degrade in motility. Referring to (iii) Competition, note that the causal nature of fitness/awareness (as one wishes to interpret it) informs a Darwinian survival mechanism. 


\section{Quale and simulations.}

3.1. Colonial awareness, a quale defined, migration, mirroring, self, intentionality. We take the cooperative, competitive development of the colony (described in Section 2.3) to represent a colony-wide awareness of its nutrient supply. In particular, we take

$$
A(x, t)=\sum_{\text {bacteria at } x} a(t)
$$

as the awareness density token (at location $x$ ) of the colony. Unlike $a(t), A(x, t)$ is not taken as binary valued $( \pm 1)$, since we anticipate a need for gradations in awareness at the colony level. Since units of awareness are unknown, we describe it in the most general mathematical terms, namely, that of a set. Then we write the colonial awareness density construct as

$$
\hat{A}(x, t)=\bigcup_{\text {bacteria at } x} \hat{a}(t),
$$

an equation without independent meaning, indicating that awareness is not necessarily a quantitative variable that might be summed, say. It also allows us to write $\hat{A}(x, t)=\varnothing$ (the empty set) for indicating when there is no awareness.

Characterization of the $\bigcup$-symbol for combining awareness is an open problem. For an indication of a solution, see the discussion of how awareness/consciousness agglomerates in neural networks in Miranker ([26]). With the constructs $A(x, t)$ and $\hat{A}(x, t)$, the colony can be viewed as functioning as a multi-cellular organism as it in fact does in quorum sensing (Waters, Bassler ([26])). We expect the colony to migrate towards its nutrient supply in the sense that $p(x, t)$ will develop so as to approximate $n(x, t)$, up to a scale factor. We describe this population profiling as a mirroring of nutrient, that is, a mirroring of $n(x, t)$ by $p(x, t) 9$ Then we interpret $p(x, t)$ as a physical quantity associated to the colonial awareness density, $\hat{A}(x, t)$. We shall view $p(x, t)$ and $\hat{A}(x, t)$ as dual, $p(x, t)$ being an externally observable quantity, $\hat{A}(x, t)$ an externally unobservable quality. With this convention, we say that $\hat{A}(x, t) / p(x, t)$ are sensations/sensors of nutrient that at the level of response, corresponding to human perception, we might by way of example call aroma/olfaction. That is, the awareness $\hat{A}(x, t)$ is an experiencing of its dual $p(x, t)$, in turn, of $n(x, t)$ (the latter mirrored by $p(x, t)$ ). These observations posit that a quale density is a pair $(p(x, t), \hat{A}(x, t))$, where $p(x, t)$ is a particular token of the quale and $\hat{A}(x, t)$ is the experiencing of it. We summarize these features in the schematic (3.3), where for clarity the term density is omitted. If the dualist features (the terms with

${ }^{9}$ In one dimension, the rotation matrix $R(\theta, \varphi)$ and the unit vector $z$ may be discarded, and the mirroring can be demonstrated analytically. In this case, the equation of motion (2.3) becomes

$$
x(t+1)=x(t)+\alpha g(t) \Delta n .
$$

To demonstrate the mirroring, rewrite this equation, using (2.8), as

$$
\Delta x=\alpha[a(t)+g(t-1)] \Delta n .
$$

Since the time average $\bar{g}=0$, time averaging this last expression for $\Delta x$ gives

$$
\overline{\Delta x}=\alpha a(t) \overline{\Delta n} \text {. }
$$

Since the fit bacteria $(a(t)=1)$ tend to survive, this expression for $\overline{\Delta x}$ indicates that bacterial displacements and the average of nutrient concentration changes $\overline{\Delta n}$ tend to proportionality. 
hats) are denied the bacteria, they become so-called zombie bacteria.

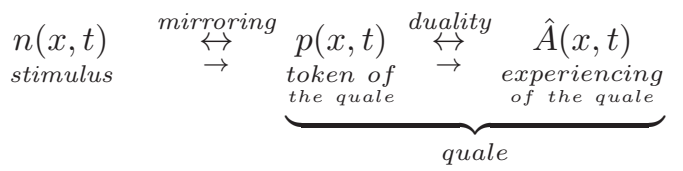

Self and intentionality: The model may also be interpreted, showing that notions of the self and intentionality also emerge. See Miranker (25) for such details.

3.2. Simulations. We now give the results of simulation 10 of the colonial behavior that demonstrate the mirroring (the basic colonial awareness response) developed in Section 3.1. We take the nutrient bath to be a rectangular pixilated region. A subset $C$ of pixels is specified, each such pixel steadily receiving a greater exogenous supply of nutrient than pixels not in $C$. Let

$$
m_{1}=\frac{\sum_{\text {pixels } \in C} p(\text { pixel })}{\sum_{\text {pixels } \in \text { bath }} p(\text { pixel })},
$$

and let

$$
m_{2}=\frac{\text { fitin }}{\text { fitout }}
$$

where

$$
\text { fitin } / \text { fitout }=\frac{\sum_{\text {pixels } \in C} \mid \text { bacterium } i \text { at pixel, } a(i)>-1 \mid}{\sum_{\text {pixels } \in / \notin C} p(\text { pixel })} .
$$

In (3.6), - denotes the number of elements in the collection specified within the bars. $m_{1}$ grades the mirroring of the exogenous nutrient supply by the colony population (symbolized by $p(x, t) \propto n(x, t)$ ), while $m_{2}$ grades the mirroring of the exogenous nutrient supply by the colonial fitness (symbolized by $A(x, t) \propto n(x, t)$ ). The nutrient bath consisting of $m \times n$ cells is populated randomly with $p \ll m \times n$ bacteria. Allowing each bacterium to undergo a single move phase (via (2.5)) initializes the simulation. Afterwards each bacterium undergoes the feed, reproduce/die, and move phases, in that order, except that the move phase is preceded by an injection of nutrient flux, $n_{e}(x, t)$, into each pixel. This full cycle of phases constitutes one time unit. The injection of nutrient flux is specified as follows:

$$
n_{e}(x, t)= \begin{cases}k \beta, & \text { if } x \in C, \\ \beta, & \text { otherwise. }\end{cases}
$$

Here $k>1.0$ is a scaling factor. $C$ is a centered subrectangle of the bath and is a quarter of its area. Finally the values of $\alpha$ (see (2.3)) and $v$ (see (2.11)) are specified at the beginning of each simulation. Ten simulations of every variant are performed and averages calculated.

Table 3.1 shows the results of testing for mirroring \# 1 of the exogenous nutrient supply by the bacterial population, i.e., of $m_{1}((3.4))$ and of testing for the mirroring \#2 of the exogenous nutrient supply by the colonial fitness, i.e., of $m_{2}((3.5))$.

\footnotetext{
${ }^{10}$ More details about these simulations can be found in the preprint by Barash, Miranker ([2]).
} 
TABLE 3.1. Mirroring effects as a function of $\alpha$ and $v$ values

\begin{tabular}{r|l|l|l|}
\cline { 2 - 4 }$\alpha$ & 0.1 & 0.5 & 1.0 \\
\hline$m_{1}$ & 0.55 & 0.25 & 0.15 \\
\hline$m_{2}$ & 1.1 & 1.9 & 2.0 \\
\hline
\end{tabular}

\begin{tabular}{r|l|l|l|}
\cline { 2 - 4 }$v$ & -3.0 & -1.0 & 1.0 \\
\hline$m_{1}$ & 0.28 & 0.57 & 0.13 \\
\hline$m_{2}$ & 1.8 & 1.2 & 2.0 \\
\hline
\end{tabular}

Finally three snapshots taken of the colony at the end of three different simulations are shown in Figure 3.2 (corresponding from left to right to the values $v=1.0,-1.0$, and 0.0 , resp.). In each case, $m=n=30, p=100$ bacteria, and $\alpha=1$. White, light gray and black pixels represent concentrations of nutrient is ascending order. Dark gray pixels represent living bacteria.
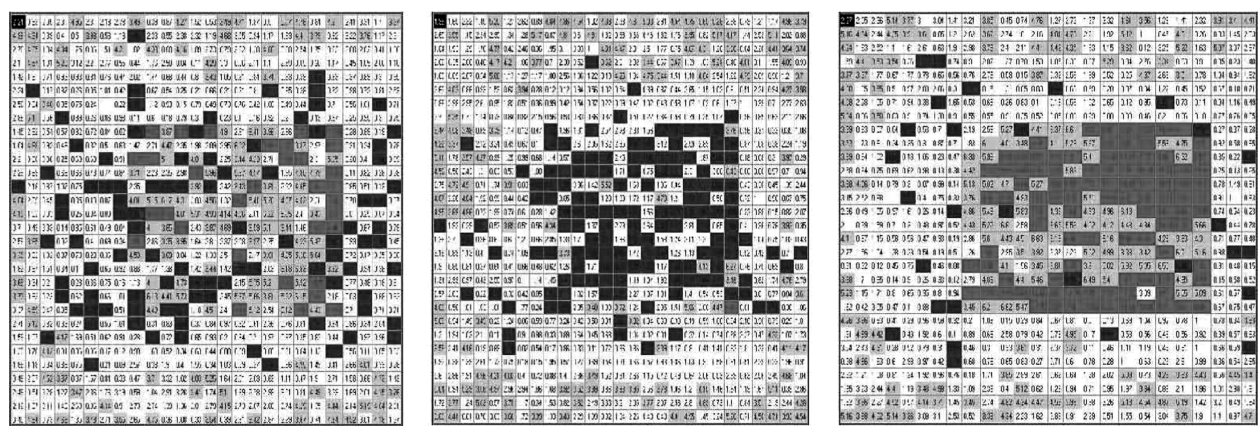

FiguRE 3.2. Bacterial sensitivity to nutrient location for different values of $v$.

Since the nutrient rich region $C$ occupies a quarter of the bath, the mirroring effect $\# 1 / \# 2$ can be said to have occurred if $m_{1}>0.25 / m_{2}>1.0$ (that is, if the bacteria have migrated into $C$ ). From Table 3.1 we see that effect \# 1 is validated only for certain parameter values, while effect \#2 is validated for all parameter choices. Also note that increasing $\alpha$ results in decreasing mirroring. We may interpret this by saying that over sensitivity to local nutrient changes is counterproductive. We also note that $m_{1}$ is very sensitive to variations in $v$, with mirroring occurring near a single value $(v=-1.0)$ and falling off rapidly with changes in either direction. The snapshots of Figure 3.2 serve as visual confirmation of this feature. These observations show that mirroring (colonial awareness) and its survival advantages are available and optimized for appropriately selected parameter values, values likely delivered in nature by Darwinian evolutionary effects.

\section{Part II: Consciousness in a neuronal assembly.}

\section{The Neuronal model.}

Letting $w$ denote the neuron's vector of afferent synaptic weights, a bacteria's position $x$ in physical space is replaced by a neuron's location $w$ in synaptic weight space, $W$. The dimension of $W$, corresponding to the largest number of afferents of any single neuron 
in the assembly, is at least as large as 200,000 in the human brain. This increase of five orders in dimension magnitude begins to suggest the range of complexity of human consciousness.

4.1. Information flux. Neurons do not forage about in physical space, but they do change their processing behavior as they change location in $W$. For clarity we employ binary-valued neural activity. Neurons may be viewed as consumers of neural transmitters, a process that takes place in the synaptic cleft. This process is implicated in information processing 11 Neurons can become better/poorer consumers of transmitter flux depending on their position in $W$, and better/poorer producers of this flux as well (i.e., more/less fit, as we shall see). Consuming flux is one way that a neuron measures information, so a neuron's motion in $W$ can be viewed as its response to information measurements.

4.2. The neuronal chromosome, synaptic weight dynamics, proto-consciousness, its token. Let $N_{i}(w)=N_{i}(w, t)$ denote the $i$ th neuron at $w \in W$, taken in some order. Set

$$
h_{i}(w, t)= \begin{cases}1, & \text { if } N_{i}(w, t) \text { fires } \\ 0, & \text { otherwise }\end{cases}
$$

Each afferent synapse may be viewed as consuming a measure of transmitter flux, and each neuronal firing, counting its axonal arborization, may be viewed as an augmentation of the transmitter (the neuronal assembly analog of nutrient) supply. Let $n_{i}(w, t)$ be the number of afferents of $N_{i}(w)$ receiving endogenous (of the assembly) signal, and let $n(w, t)=\sum_{i: \text { neurons at } w} n_{i}(w, t)$. Then the assembly's transmitter flux dynamics, the neuronal feature corresponding to bacterial nutrient dynamics in (2.6), are

$$
n(w, t+1)=\sum_{i: \text { neurons at } w} h_{i} K_{i}(w, t)-n(w, t)+n_{e}(w, t) .
$$

Here $K_{i}(w, t)$ is a multiplier representing the degree of axonal arborization of $N_{i}(w)$, and $n_{e}(w, t)$ is the number of afferents of neurons at $w$ receiving exogenous (from outside the assembly) signals. The sum in (4.2) represents production of transmitter flux. So it is the firing neurons themselves that inform the neural transmitter ("nutrient") supply. Next take

$$
a_{i}(w, t)=\operatorname{sgn}\left[\frac{n_{i}(w, t)}{n_{i}(w, t-1)}-1\right], \quad \forall i
$$

for the fitness of $N_{i}(w)$ (see (2.7)). That is, neuron $N_{i}(w)$ is fit/not-fit at $w$ at time $t$ (i.e., $\left.a_{i}(w, t)= \pm 1\right)$ ) if it improves/degrades in ability to acquire input-flux, according to the flux dynamics in (4.2). This fitness quality is informed by the neuron's location in $W$ as (4.3) shows. Neuronal fitness does not supervene solely on the neuron. It also depends on the neuron's environment (connectivity arrangements and activity of connecting neurons). The measurable physical quantity $a_{i}(w, t)$ in (4.3) is the token of the neuronal consciousness, and the corresponding dualist construct, $\hat{a}(x, t)$, that we

\footnotetext{
${ }^{11}$ Substances called auto-inducers (Ben Jacob et al. [3]) might be viewed as the colonial counterparts of neural transmitters. They are emitted by bacteria and diffuse. It is the concentration of auto-inducers detected in turn by the bacteria that provides communication between them. We thank an anonymous referee for pointing out that the immune system supplies an analogous feature.
} 
shall call the neuronal proto-consciousness is the observer process of the measurement and computation represented by (4.3).

Neuronal chromosome. The model neuron possesses a feature that can perform the function of a virtual gene such as the $g$ of Section 2.1. This intrinsic feature is the vector $w$ of its afferent synaptic weights, which we shall regard as a virtual neuronal chromosome. Each individual synaptic weight can be viewed as playing the role of a gene in that chromosome. (This virtual chromosome must not be confused with any of the neuron's literal genomic material.) Hebb's law is the customary choice for the $w(t)$ dynamics (Haykin [14]). Then we use it, employing the token $a_{i}(w, t)$ as a scaling factor, as follows:

$$
w_{i}(t+1)=w(t)+a_{i}(w, t) H\left(v_{i}^{a}(t), v_{i}^{e}(t+1)\right)+v \chi, \quad \forall i
$$

See the winning neuron specification in the following Section 4.3 for motivation for the scaling of $H$ by $a_{i}(w, t)$ in (4.4). The Hebb function (Hebb ([15, 16])),

$$
H=H\left(v^{a}(t), v^{e}(t+1)\right)
$$

determines the correlation of each synaptic input with the neuronal output. In (4.5), $v^{a}(t)$ is the vector of neuronal afferents (voltages that are approximately proportional to the transmitter flux arriving at an afferent), and the scalar $v^{e}(t+1)$ models that neuron's efferent. The quantities $a_{i}(w, t)$ and $H\left(v_{i}^{a}(t), v_{i}^{e}(t+1)\right)$ refer to neuron $N_{i}(w)$. The quantity $w_{i}(t+1)$ is the subsequent location of $N_{i}(w)$ in $W$. The quantity $v \chi$ (with scaling factor $v$ and random variable $\chi$ ) representing noise can be viewed as a stochastic mutation generating term. If $v \chi<0$, it is called a forgetting factor in neuronal modeling (Haykin [14). So we see that the dynamics (4.4) in $W$ are the neuronal analogs of the bacterial dynamics (2.1)-(2.5).

4.3. Population dynamics. Population dynamics have a more complex character in the neural assembly than in the bacterial case. The analog of $p(x, t)$ is $p(w, t)$, the number of neurons at a point $w$ in $W$ that have fired at time $t$. The three features: motion, reproduction and competition of Section 2 drive the changes in $p(w, t)$ as well, with the modification that $(i)$ competition in the colony for a bacterium to reproduce is replaced by competition in the assembly for the opportunity of a neuron to fire and ( $i i)$ the ability to consume nutrient in the colony is replaced by the ability to consume transmitter flux. The competition among neurons in $(i)$ is implemented by lateral inhibition, for example. The ability in (ii) to consume is location dependent (in physical space for the colony or in synaptic weight space $W$ for the assembly). Unlike the colonial case, neurons that don't fire, while not contributing to the current value of $p(w, t)$, are not eliminated from the model. Such neurons await a further opportunity to participate. There is still a survival of the fittest aspect to be described presently.

Population dynamics are defined in terms of so-called winning neurons, namely

$$
p(w, t)=\# \text { of neurons at } w \text { specified as winners at time } t .
$$


Winning neuron specification. If at least one neuron fires, the transmitter flux per firing neuron at $w$ at time $t$ is

$$
s=s(w, t)= \begin{cases}0, & \text { if no neuron fires }, \\ \frac{\sum_{i} n_{i}}{\sum_{i} h_{i}}, & \text { otherwise. }\end{cases}
$$

Let $w_{j}=w_{j}(t)=\left(w_{j 1}, w_{j 2}, \ldots\right)$ be the afferent synaptic weight vector of $N_{j}(w)$. There is only $s$ amount of flux per firing neuron. Since our model neurons are binary, the $\sum w_{i j}$ taken over $\lfloor s\rfloor$ particular afferents of a neuron is its typical total input activity. Let $s_{j}$ be the random variable that takes the values

$$
s_{j}=\sum_{i}^{(s)} w_{j i}
$$

where the sum is over a collection of $\lfloor s\rfloor$ of the components of $w_{j}$ chosen randomly from all of these components. Let $\bar{s}_{j}$ denote the mean value of $s_{j}$, that is, the mean (at time $t$ ) of the total input activity of $N_{j}(w)$. If

$$
\bar{s}_{j}>\theta_{j},
$$

where $\theta_{j}$ is the firing threshold of $N_{j}(w)$, then the latter is a winning neuron. Neurons that fail the test in (4.9) are losers. The heuristic is that during a sustained epoch of neuro-processing, a neuron that satisfies (4.9) is expected to fire more frequently than a neuron that does not. The fitness $a_{i}(w, t)$ expresses the tendency of a neuron to fire and so should (algebraically) scale the change in $w_{i}(t)$ as indicated in (4.4).

Natural selection. The neuronal features just described characterize a Darwinian [8] process, since fitness scales a neuron's ability to fire (to be a winner) as (4.4) shows. In nature a neuron that doesn't participate (fire) for too long a period may atrophy and die. Although inessential for our present purposes, such a refinement would give these features a 'survival of the fittest' character. Genetic mutation, Weismann's addendum to Darwin's ideas (Harti, Jones [13]) can be adapted into the genes and chromosomes of our neuronal model using $v \chi$ in (4.4) (abrupt random changes in synaptic weights).

\section{Qualia and applications.}

5.1. Assembly awareness, a quale defined, mirroring. Let

$$
A(w, t)=\sum_{i: \text { neurons at } w} a_{i}(w, t)
$$

(compare (3.1), (3.2)ff) be the token of the derived consciousness construct $\hat{A}(w, t)$, where

$$
\hat{A}(w, t)=\bigcup_{i: \text { neurons at } w} \hat{a}_{i}(w, t) .
$$

An alternative choice for the assembly awareness density token is the measurable quantity

$$
A(w, t)=\frac{n(w, t)}{n(w, t-1)}-1 .
$$

We shall take $\hat{A}(w, t) \neq \varnothing$ only if its token $A(w, t)>0$.

We take the number of winning neurons, $p(w, t)$, to be a measurable quantity dual to an assembly consciousness density $\hat{A}(w, t)$. Up to a scale factor, we expect $p(w, t)$ to mirror 
the transmitter flux $n(w, t)$. That is, the number of winning neurons should reflect the intensity of the neuronal activity (the quantity of transmitter flux). (Compare with the mirroring measures $m_{1}$ and $m_{2}$ of the simulation in Section 3.2.) As a physical quantity, $p(w, t)$ is measurable but unconscious (see (4.6)ff), while the derived construct $\hat{A}(w, t)$ is externally unobservable (say, a proto-consciousness density). With this convention, we say that $\hat{A}(w, t)$ (proto-conscious) $/ p(w, t)$ (unconscious) are sensations/sensors of the encoded information being processed by the neuronal assembly. That information is conventionally specified in terms of the assembly's collection of action potentials (the collection of efferents $v^{e}$ appearing in (4.5)), and these, in turn, are each approximately proportional to a quantity of transmitter flux. We posit that $\hat{A}(w, t)$ is an experiencing of its dual $p(w, t)$. That is, $\hat{A}(w, t)$ is an experiencing of an information density encoded and being processed by the subassembly of neurons at $w \in W$. The pair $(p(w, t), \hat{A}(w, t))$ is the quale density corresponding in turn to a stimulus $n(w, t)$, the latter mirrored by $p(w, t)$ (see the schematic (3.3)). In this neuronal assembly case, the stimulus could be conveyed in whole or in part from another neuronal assembly, perhaps one corresponding to a sense organ.

5.2. Applications. The applications to be considered model the presence or absence of consciousness, and if present they specify its location (e.g., pain in the finger tip, vision out there in space...). Since it is the sign of the token corresponding to a neuronal structure that specifies this (see the definition in Section 2.2), applications are developed through consideration of such a neuronal structure along with the derivation of the mathematical expression of its associated token. The human mind is unable to generate certain qualia in the absence of (exogenous) sensory input, for example, unable to create the smell of a rose without a rose being present. That is, sensory input is necessary for experiencing such a quale. We begin by interpolating an argument in Section 5.2.1, demonstrating this effect in the context of the bacterial colony, an application of the bacterial level construct of Part I that serves as a model of more extensive applications for the neuronal assemblies that follow in Section 5.2.2.

5.2.1. A colonial application. To show that awareness vanishes in the absence of sensory input, set $n_{e}(x, t)=0$. Then using (2.6), the bracketed term in (2.7) can be written as

$$
\frac{\bar{n}(x, t)-\beta \bar{p}(x, t)}{\bar{n}(x, t)}-1=-\beta \frac{\bar{p}(x, t)}{\bar{n}(x, t)} .
$$

By definition (see Section 2.2), colonial awareness/perception disappears $(\hat{A}=\varnothing)$, since the members of (5.4) are negative.

5.2.2. Neuronal assembly applications. We now develop the neuronal assembly applications listed at the end of Section 1.2.

\#1. Conditions for generating qualia, a threshold effect

This is an adaptation of the colonial application to the neuronal assembly, and so is relevant to human consciousness. Namely, sensory input is a necessary condition for the experiencing of certain qualia 12 We proceed as in Section 5.2.1, setting $n_{e}(w, t)=0$.

\footnotetext{
${ }^{12}$ Compare Kant [17], "All our knowledge begins with the senses, proceeds then to understanding and ends with reason... It is beyond a doubt that all our knowledge begins with experience."
} 
Then combining (4.2) and (5.3),

$$
A(w, t)=\frac{\sum_{j: \text { neurons at } w} h_{j} K_{j}(w, t-1)}{n(w, t-1)}-2 .
$$

Then define the consciousness token of the entire assembly at time $t$ to be

$$
A(t)=\sum_{w} A(w, t)
$$

the sum taken over the finite set of $w$ specified by the assembly. Assembly consciousness is (see (5.2) for interpretation)

$$
\hat{A}(t)=\bigcup_{w} \hat{A}(w, t) .
$$

The numerator in (5.5) represents all of the neural transmitter flux produced by the assembly at $t-1$. The denominator represents that flux plus exogenous flux entering the assembly at time $t-1$. Then the fraction in (5.5) is less than or equal to one, and so, $A(w, t) \leq-1$. Then in the absence of exogenous (say, sensory) input, $\hat{\mathbf{A}}(t)=\varnothing$. Moreover to experience a quale, the input must be present, and the ratio $n_{e}(w, t-$ $1) / n(w, t-1)$ must exceed $A(w, t)$ given in (5.5), the latter requirement being a threshold effect.

\section{\#2. Qualia are located in the peripheral nervous system}

For this application that informs olfaction, gustation and haptic response, we employ a three-layer compartmental model of the nervous system. Layer 0 is the sensory input layer. Layer 2 is the brain. Layer 1 represents the remainder, the peripheral nervous system and the cord. So layer 0 feeds layer 1 , and layer 1 interacts reciprocally with layer 2. To reflect these three compartments, (4.2) is replaced by

$$
\begin{aligned}
n^{(j)}(w, t+1)= & \sum_{i: \text { neurons at } w} h_{i}^{j} \lambda_{i}^{j}(w, t)-n^{(j)}(w, t)+(2-j) n^{(0)}(w, t) \\
& +\sum_{i: \text { neurons at } w} h_{i}^{j} K_{i}^{j k}(w, t), j, k=1,2, j \neq k .
\end{aligned}
$$

Here $n^{(0)}(w, t)$ is the sensory input from layer 0 to layer $1 . n^{(j)}(w, t)$ is the number of afferents in layer $j, j=1,2$, receiving input. Corresponding to layer $k, k=1,2, K_{i}^{j k}(w, t)$ is a multiplier representing the part of the axonal arborization of the $i$ th neuron at $w$ that enervates a neuron in layer $j, j=1,2, j \neq k . \quad \lambda_{i}^{j}(w, t)$ corresponds analogously to lateral enervation within layer $j$. Then $h_{i}^{j}=1 / 0$, if in layer $j, N_{i}(w)$ fires/not-fires, respectively. Correspondingly, (5.3) is replaced by

$$
A^{(j)}(w, t)=\frac{n^{(j)}(w, t)}{n^{(j)}(w, t-1)}-1, \quad j=1,2 .
$$

Replacing $t+1$ by $t$ in (5.8) and inserting the result into (5.9), the latter becomes

$$
A^{(j)}(w, t)=\frac{\Phi^{j k}(w, t-1)+(2-j) n^{(0)}(w, t-1)}{n^{(j)}(w, t-1)}-2, \quad j, k=1,2, j \neq k,
$$

where

$$
\Phi^{j k}(w, t-1)=\sum_{i: \text { neurons at } w}\left[h_{i}^{j}\left(\lambda_{i}^{j}(w, t-1)+K_{i}^{j k}(w, t-1)\right)\right] .
$$


The term $B=\Phi^{j k}(w, t-1) / n^{(j)}(w, t-1)$ from (5.10) is smaller than unity (for reasons analogous to those in the paragraph following $(5.7))$, so $A^{(j)}(w, t)$ is negative unless the term $(2-j) n^{(0)}(w, t-1) n^{(j)}(w, t-1)$ survives and dominates $B$. Survival occurs only for $j=1$ (for layer 1 ), and dominance occurs when $n^{(0)}(w, t)$ is sufficiently large (when there is adequate sensory input). Thus the consciousness $\hat{A}(w, t) \neq \varnothing$ only in the peripheral nervous system $(j=1)$ and then only in the presence of adequately strong sensory input, a threshold effect.

\#3. Visual and auditory imagery located out in space (the environment)

This application is a corollary of \#2, so we proceed by identifying the three layers in question, not all of which are corporeal (as we shall see). We discuss the case of vision only, since the changes for audition (e.g., eye replaced by inner ear) are straightforward.

Layer 0 is the light source that supplies light flux (photons). Layer 1 consists of three compartments. The first of these consists of the objects seen 13 The flux from layer 0 is processed by absorption in and reflection from the first compartment, and the resulting flux (light stream) is passed on to the second compartment, the eye. The eye processes the flux from the first compartment by focusing it on the retina, which, after preliminary processing in the retina, passes flux (action potentials) via the optic nerve to the LGN, the third compartment. Layer 2 is the rest of the visual cortex that interacts reciprocally with the LGN.

Referring to \#2, we see that the consciousness corresponding to vision occurs in layer 1 whose first compartment consists of the objects seen. Vision makes use of a mix of structure and flux types. The structures are neuronal, corporeal (but not neuronal) and extra-corporeal. The nutrient flux changes from streams of photons (proceeding first to and then from layer 0 and through the front of the eye) to the customary neural transmitter. The processing of the flux is perforce disparate. This suggests that consciousness supervenes not only on the neuronal and non-neuronal corpus, but of the environment as well. As noted in Section 4.2, the environment's role in perception is more substantive than as a provider of input. While this last remark applies to vision and audition, it might very well apply to other senses. It seems to be relevant to the perception of heat by pit vipers, the perception of electrical waves to certain eels, and perhaps the perception of gravity or magnetic fields to migratory birds.

Model complexity: Layer 2 represents neuronal processing of more than 20 known richly enervated reciprocally connected visual brain regions. It is not merely a principle of economy in the model (Ockham) that permits this collapse of 20 into one. Indeed examining the details of (5.8)-(5.10) in \#2 based on which of the vision arguments are made, we could accommodate these 20 such regions by appending additional layers to the model, and correspondingly, replacing $j$ and/or $k=1,2$ by $j$ and/or $k=1, \ldots, 21$ in (5.8)-(5.10). The conclusion would be the same. Namely, what was called survival in the last paragraph of \#2 still occurs only for $j=1$. That is, consciousness occurs only in layer one (i.e., 'out there'). There is no consciousness perceived in any of the newly introduced layers.

\footnotetext{
${ }^{13}$ The use of the environment as a working store for cognitive processing of visual information is an idea explored by Ballard, Hayhoe, Pook, and Rao [1.
} 


\section{\#4. Dream imagery is located in a virtual space}

As in application \#2, we proceed by describing the three layers in the dream imagery model. These are three different parts of the brain: 1) a source of imagery (from memory, say dream memory), 2) an intermediate structure where the consciousness of the imagery is manifest, and 3) the remainder of the brain. That is, we postulate that a sufficiently complex brain is able to simulate a visual sensory system (say as described in \#2) endogenously. So the location of these dream images is in the intermediate structure. Not being able to identify a physical location for the intermediate structure, we perceive it to be in some virtual space.

\section{\#5. Hallucinations, in particular phantom pain}

Hallucinations are pathological phenomena. An explanation of them flows when certain variables in our model behave pathologically. We illustrate this in the case of phantom pain.

Referring to Section 5.2.3, to accommodate the missing limb, layer 0 and part of layer 1 are absent. Then set $n^{(0)}(w, t-1)=0, j=1$ and $k=2$ in (5.10), giving

$$
A^{(1)}(w, t)=\frac{\sum_{i: \text { neurons at } w}\left[h_{i}^{1}\left(\lambda_{i}^{j}(w, t-1)+K_{i}^{12}(w, t-1)\right)\right]}{n^{(1)}(w, t-1)}-2 .
$$

In application $\# 2$, we argued that this quantity is negative (in fact, $A^{(1)}(w, t) \leq-1$ ). So under normative conditions, there is no perceptual awareness in layer 1 (in the part of it that is missing). That is, there is no phantom pain. Phantom pain has been shown to result from abnormally high levels of Nav1.3, a specific sodium channel in thalamic neurons, causing in turn, a cortical stimulation (Waxman [37]). These events are layer 2 events in our model, which we shall characterize as a pathological augmented creation of neurotransmitter flux in layer 2. This flux stimulates layer 1 in turn. That is, these events are characterized in our model as a pathological and excessively high value of $K_{i}^{12}$ in (5.12). If this value is large enough, (5.12) shows that $A^{(1)}(w, t)$ will be positive, so there is a perception $\left(\hat{A}^{(1)}(w, t) \neq \varnothing\right)$ of (phantom) pain in the missing part of layer 1 . Compare this outside the body perception with the location of the 'out there' perception of vision described in \#3.

\section{\#6. Pheromones}

This is also a corollary, one directly of (5.10). The argument following (5.10) shows that awareness of a sensory input can only occur in layer 1 , but then only in the presence of adequately strong input. Adequate means that the relative input satisfies the inequality

$$
\frac{n^{(0)}(w, t)}{n^{(1)}(w, t)}>2
$$

This inequality is derived by setting $j=1$ in the term $(2-j) \frac{n^{(0)}(w, t)}{n^{(j)}(w, t)}$ in $(5.10)$.

Then we posit that an unconscious sense (such as a response to a pheromone) corresponds to a neuronal structure that is wired up so that the inequality in (5.13) is never satisfied. This indicates that it is a limitation of the associated circuitry of layer 1 and/or its activity, which inhibits consciousness of pheromones. 
5.3. Evolution. The argument in \#6 on pheromones suggests how perceptual ability might be an evolving, alternatively a deteriorating property; appearing (strengthening even to consciousness) as the relevant neural circuit/activity becomes more robust, alternatively evanescing as that circuit/activity degenerates. Referring to \#3 on vision, we see that alterations in the environment on an evolutionary scale might also come into play in this evolving aspect of consciousness. An example of deterioration where sighted creatures that convert to a lightless environment evolve sightless descendents is supplied by Axtyanax fasciatus mexicanus (blind cave fish). Brain injuries furnish examples on the scale of a lifetime (Sacks 29])).

\section{REFERENCES}

[1] Ballard, D., Hayhoe, M., Pook, P., Rao, R. (2001). Deictic codes for the embodiment of cognition, Behavioral and Brain Sciences.

[2] Barash, V., Miranker, W. (2006). Modeling awareness in a bacterial colony, Yale DCS TR 1369.

[3] Ben Jacob, E., Becker, I., Shapira, Y., Levine, H. (2004). Bacterial linguistic communication and social intelligence, Trends in Microbiology, 12, 366-372.

[4] Chalmers, D. (1996). The Conscious Mind: In Search of a Fundamental Theory, NY, Oxford.

[5] Churchland, P. M. (1984). Matter and Consciousness, Cambridge, MIT Press.

[6] Curtis, H., Barnes, N.S. (1989). Biology, New York, Worth Publishers.

[7] Damasio, A. (2003). Looking for Spinoza, New York, Harcourt.

[8] Darwin, C. (1859). The Origin of Species by Means of Natural Selection.

[9] Dawkins, R. (1976). The Selfish Gene, Oxford University Press.

[10] Dennett, D. (1991). Consciousness Explained, Boston, Little Brown.

[11] Dennett, D. (1996). Kinds of Minds, Towards Understanding Consciousness, NY, Basic.

[12] Edelman, G., Tononi, G. (2001). A Universe of Consciousness: How Matter Becomes Imagination, New York, Basic Books.

[13] Harti, D., Jones, E. (2005). Genetics: Analysis of Genes and Genones, Jones \& Bartlett, Boston.

[14] Haykin, S. (1999). Neural Networks, a Comp. Foundation, Prentice-Hall, Upper Saddle River.

[15] Hebb, D. (1946). On the nature of fear, Physiol. Rev. 53, 259-276.

[16] Hebb, D. (1949). Organization of Behavior: A Neurophysiological Theory, New York, Wiley.

[17] Kant, I. (1781), Critique of Pure Reason

[18] Llinas, R. (2001). $i$ of the Vortex, Cambridge, MIT Press.

[19] Margenau, H. (1950). Nature of Physical Reality; A Philosophy of Modern Physics, NY, McGrawHill. MR0035258 (11:709h)

[20] Margenau, H. (1978). Physics and Philosophy: Selected Essays, Reality; a Philosophy of Modern Physics, Boston, Dordrecht. MR 529767 (80c:01020)

[21] Margulis, L. (2001). The Conscious Cell, Annals of the New York Academy of Sciences. 929.

[22] Mayr, E. (1982). The growth of biological thought. Cambridge, Harvard University Press.

[23] McDermott, D. (2001). Mind and Mechanism, Cambridge, MIT Press.

[24] Miranker, W. (2000). Consciousness is an Information State, J. Neural Parallel and Scientific Computation, 8, 83-104.

[25] Miranker, W.L. (2005) Consciousness, a Darwinian Process, Yale DCS TR 1344.

[26] Miranker, W.L. (2008). The neural network as a renormalizer of information, Quart. Appl. Math. 66, 379-394. MR2416779 (2009b:91127)

[27] Nagel, T. (1974), What is it like to be a bat? Philosophical Review 83, 435-450.

[28] Penrose, R. (1994). Shadows of the Mind, New York, Oxford University Press. MR1865778 (2002g:00003)

[29] Sacks, O. (1995). An Anthropologist on Mars, New York, Knopf.

[30] Schrödinger, E. (1958). Mind and Matter, Cambridge University Press.

[31] Sheets-Johnstone, M. (1998). Consciousness; a Natural History, J. Consciousness Studies 3, 260294.

[32] Stapp, H. (1998). The hard problem: a quantum approach, J. Consciousness Studies, 3, 192-210.

[33] Stapp, H. (1999). Attention, intention, and will in quantum physics, J. Consciousness Studies. 
[34] Turing, A.M. (1952). The chemical basis of morphogenesis. Phil. Trans. Royal Soc. London B237, 37-72.

[35] Velmans, M. (2000). Understanding Consciousness, London, Routledge.

[36] Waters, C.M., Bassler, B.L. (2005). Quorum Sensing: Cell-to-cell communication in bacteria, Annual Review of Cell and Developmental Biology, 21, 319-346.

[37] Waxman, S. (2005). Brain (August 18, 2005, online). 